\title{
GENERALISED DIRICHLET SERIES AND HECKE'S FUNCTIONAL EQUATION $\dagger$
}

\author{
by BRUCE C. BERNDT \\ (Received 7th February 1967)
}

\section{Introduction}

The generalised zeta-function $\zeta(s, a)$ is defined by

$$
\zeta(s, a)=\sum_{n=0}^{\infty}(a+n)^{-s},
$$

where $a>0$ and $\operatorname{Re} s>1$. Clearly, $\zeta(s, 1)=\zeta(s)$, where $\zeta(s)$ denotes the Riemann zeta-function. In this paper we consider a general class of Dirichlet series satisfying a functional equation similar to that of $\zeta(s)$. If $\phi(s)$ is such a series, we analogously define $\phi(s, a)$. We shall derive a representation for $\phi(s, a)$ which will be valid in the entire complex $s$-plane. From this representation we determine some simple properties of $\phi(s, a)$.

Throughout the sequel we let $s=\sigma+i t$ and $z=x+i y$ with $\sigma, t, x$ and $y$ real. If $c$ is real, we denote the integral $\int_{c-i \infty}^{c+i \infty}$ by $\int_{(c)}$. The summation sign $\Sigma$ appearing with no indices will always mean $\sum_{n=1}^{\infty}$.

The following definition is essentially that of Chandrasekharan and Narasimhan (1).

Definition 1. Let $\left\{\lambda_{n}\right\}$ and $\left\{\mu_{n}\right\}$ be two sequences of positive numbers tending to $\infty$, and $\{a(n)\}$ and $\{b(n)\}$ two sequences of complex numbers not identically zero. Consider the functions $\phi$ and $\psi$ representable as Dirichlet series

$$
\phi(s)=\Sigma a(n) \lambda_{n}^{-s}, \quad \psi(s)=\Sigma b(n) \mu_{n}^{-s}
$$

with finite abscissae of absolute convergence $\sigma_{a}$ and $\sigma_{a}^{*}$, respectively. If $r$ is real, we say that $\phi$ and $\psi$ satisfy the functional equation

$$
\Gamma(s) \phi(s)=\Gamma(r-s) \psi(r-s)
$$

if there exists in the $s$-plane a domain $D$ which is the exterior of a bounded closed set $S$ such that in $D$ a holomorphic function $\chi(s)$ exists with these properties:

(i) $\chi(s)=\Gamma(s) \phi(s),\left(\sigma>\sigma_{a}\right)$,

$$
\chi(s)=\Gamma(r-s) \psi(r-s),\left(\sigma<r-\sigma_{a}^{*}\right)
$$

$\dagger$ The main result of this paper appeared in the author's Ph.D. dissertation written under the direction of Professor J. R. Smart at the University of Wisconsin in 1966. 
(ii) if $c$ and $\eta$ are chosen so that $c>\max \left(0, \sigma_{a}, \sigma_{a}^{*}\right)$, and $S$ lies outside $R=\{s: r-c<\sigma<c,|t|>\eta\}$ but in $r-c<\sigma<c$, then, for some constant $\theta<1$,

$$
\chi(s)=O\left(\exp \left[e^{\theta \pi|s| /(2 c-r)}\right]\right),
$$

uniformly in $R$ as $|s| \rightarrow \infty$.

If $b(n)=\gamma a(n)$ with $\gamma= \pm 1, r>0, \lambda_{n}=\mu_{n}=2 \pi n / \lambda$ with $\lambda>0$, and $(s-r) \phi(s)$ is entire, then $(2 \pi / \lambda)^{s} \phi(s)$ is a Dirichlet series of signature $(\lambda, r, \gamma)$ according to the definition of Hecke (2). Thus, $\zeta(2 s)$ is of signature $\left(2, \frac{1}{2}, 1\right)$.

Definition 2. If $\phi$ satisfies definition 1 , we define a generalised Dirichlet series $\phi(s, a)$ by

where $a>0$ and $\sigma>\sigma_{a}$.

$$
\phi(s, a)=\Sigma a(n)\left(a+\lambda_{n}\right)^{-s},
$$

Note that $\zeta(s, a)$ does not satisfy definition 2 .

\section{Preliminary results}

We collect here some lemmas to be employed in the sequel.

Lemma 1. We have

$$
\Gamma(s)=O\left(|t|^{\sigma-\frac{1}{2}} e^{-\pi|t| / 2}\right),
$$

uniformly for $-\infty<\sigma_{1} \leqq \sigma \leqq \sigma_{2}<\infty$, as $|t| \rightarrow \infty$.

Lemma 2. For $x>0$ and $0<c<\sigma$,

$$
\frac{1}{2 \pi i} \int_{(c)} \Gamma(z) \Gamma(s-z) x^{-z} d z=\Gamma(s) /(1+x)^{s} .
$$

This result is stated in (4), p. 192.

Lemma 3. Let $f$ be holomorphic in a strip $S$ given by $a<\sigma<b,|t|>\eta>0$, and continuous on the boundary. If for some constant $\theta<1$,

$$
f(s)=O\left(\exp \left[e^{\theta_{\pi}|s| /(b-a)}\right]\right),
$$

uniformly in $S, f(a+i t)=o(1)$ and $f(b+i t)=o(1)$ as $|t| \rightarrow \infty$, then $f(\sigma+i t)=o(1)$ uniformly in $S$ as $|t| \rightarrow \infty$.

This is a version of the Phragmén-Lindelöf theorem ((3), p. 109).

Lemma 4. Let $K_{v}(x)$ denote the usual modified Bessel function. Then,

$$
\begin{aligned}
2 K_{v}(x) & =\frac{1}{2 \pi i} \int_{(c)}(x / 2)^{v-2 s} \Gamma(s-v) \Gamma(s) d s, c>\max (0, \operatorname{Re} v) ; \\
K_{v}(x) & =0\left(x^{-\frac{1}{2}} e^{-x}\right), \text { as } x \rightarrow \infty \\
K_{v}(x) & =K_{-v}(x) \\
K_{\frac{1}{2}}(x) & =(\pi / 2 x)^{\frac{1}{2}} e^{-x} .
\end{aligned}
$$

Result (2.1) is given in (4), p. 197; (2.2), (2.3) and (2.4) are found in (5), pp. 202, 79 and 80 , respectively. We note from $(2.1)$ that $K_{v}(x)$ is an entire function of $v$. 


\section{Main results}

We now establish the representation theorem for $\phi(s, a)$.

Theorem. Let $\phi(s, a)$ denote a generalised Dirichlet series and let

$$
R(s, a)=\frac{1}{2 \pi i} \int_{C} \Gamma(s-z) \chi(z) a^{z} d z,
$$

where $C$ is a curve, or curves, chosen so that $C$ encircles all of $S$ and does not contain $s$, if possible, for a given fixed value of $s$. Then,

$$
\Gamma(s) a^{s} \phi(s, a)=2 a^{(r+s) / 2} \Sigma b(n) \mu_{n}^{(s-r) / 2} K_{r-s}\left(2 \sqrt{a \mu_{n}}\right)+R(s, a),
$$

for those values of $s$ such that the series on the right-hand side converges uniformly and which are not contained in $C$ for a suitable choice of $C$. In particular, if $2 \sqrt{a \mu_{n}} \geqq(1+\varepsilon) \log n, \varepsilon>0$, for $n \geqq N$, and the singularities of $\chi(s)$ are isolated, (3.1) is valid for all values of $s$, except these isolated singularities.

Proof. Let $c$ be given as in definition 1 and consider $s$ as fixed with $\sigma>c$. Then,

$$
\begin{aligned}
\frac{1}{2 \pi i} \int_{(c)} \Gamma(s-z) \Gamma(z) \phi(z) a^{z} d z & =\Sigma a(n) \frac{1}{2 \pi i} \int_{(c)} \Gamma(s-z) \Gamma(z)\left(\lambda_{n} / a\right)^{-z} d z \\
& =\Gamma(s) a^{s} \phi(s, a),
\end{aligned}
$$

upon an application of lemma 2. The change in order of summation and integration is justified by absolute convergence, since by lemma 1 ,

as $|y| \rightarrow \infty$.

$$
\Gamma(s-z) \Gamma(z)=O\left(|y|^{\sigma-1} e^{-\pi|y|}\right),
$$

We now move the line of integration to $r-c+i t,-\infty<t<\infty$, by integrating along the boundary of a rectangle with vertices $c \pm i T$ and $r-c \pm i T$ and then letting $T \rightarrow \infty$. By (1.1), lemma 1 and lemma 3, the integrals along the horizontal sides tend to 0 as $T \rightarrow \infty$. Hence,

where

$$
\frac{1}{2 \pi i} \int_{(c)} \Gamma(s-z) \chi(z) a^{z} d z=I(s, a)+R(s, a),
$$

$$
I(s, a)=\frac{1}{2 \pi i} \int_{(r-c)} \Gamma(s-z) \chi(z) a^{z} d z .
$$

Replacing $z$ by $r-z$, using the functional equation, and interchanging the order of summation and integration by absolute convergence, we find

$$
\begin{aligned}
I(s, a) & =a^{r} \Sigma b(n) \frac{1}{2 \pi i} \int_{(c)} \Gamma(z-\{r-s\}) \Gamma(z)\left(a \mu_{n}\right)^{-z} d z \\
& =2 a^{(r+s) / 2} \Sigma b(n) \mu_{n}^{(s-r) / 2} K_{r-s}\left(2 \sqrt{a \mu_{n}}\right)
\end{aligned}
$$


upon an application of (2.1), provided $c>\max (0, r-c)$. Combining (3.2), (3.3) and (3.4), we have established (3.1). By analytic continuation (3.1) is valid for those values of $s$ such that (3.4) converges uniformly and which are not contained in $C$. However, by (2.2)

$$
I(s, a)=O\left(\Sigma|b(n)| \mu_{n}^{\left(\sigma-r-\frac{1}{2}\right) / 2} e^{-2 \sqrt{a \mu_{n}}}\right)
$$

It is easy to see that the series of (3.5) converges uniformly if

$$
2 \sqrt{a \mu_{n}} \geqq(1+\varepsilon) \log n, \varepsilon>0 \text {, for } n \geqq N .
$$

In particular, we have

Corollary 1. Let $f(s)$ denote a Dirichlet series of signature $(\lambda, r, \gamma)$, and let $\rho$ denote the residue of $f(s)$ at $s=r$. Then, for all $s$,

$$
\begin{aligned}
f(s, a)=a^{-s} f(0)+\Gamma(r) \Gamma(s-r) a^{r-s} \rho / \Gamma(s) & \\
& +\frac{2 \gamma}{\Gamma(s)}\left(\frac{2 \pi}{\lambda}\right)^{s} \Sigma a(n)\left(\frac{a}{n}\right)^{(r-s) / 2} K_{r-s}(4 \pi \sqrt{a n} / \lambda) .
\end{aligned}
$$

Proof. The result is immediate on noting that $\chi(s)$ has at most simple poles at $s=0$ and $s=r$ and on replacing $a$ by $2 \pi a / \lambda$ in (3.1).

In the following corollaries we assume $f(s)$ is a Dirichlet series of signature $(\lambda, r, \gamma)$.

Corollary 2. If $f(s)$ is entire, $f(s, a)$ has simple zeros at $s=0,-1,-2, \ldots$. entire.

Corollary 2 is clear, since, from the functional equation, $f(0)=0$ if $f$ is

Corollary 3. If $f(s)$ is not entire and $r$ is not an integer, then

$$
f(s, a)=a^{-s} f(0), s=0,-1,-2, \ldots .
$$

Corollary 4. Suppose $f(s)$ is not entire. If $r$ is not an integer, $f(s, a)$ has simple poles at $s=r, r-1, r-2, \ldots$. If $r$ is an integer, $f(s, a)$ has simple poles at $s=1,2, \ldots, r$.

Corollary 5. $f(s, a)$ is entire if and only if $f(s)$ is entire.

\section{Examples}

Let $f(s)=\zeta(2 s)$. Since $\zeta(0)=-\frac{1}{2}$ and $\rho=\frac{1}{2}$, we have, by corollary 1 on replacing $n$ by $n^{2}$,

$$
f(s, a)=-a^{-s} / 2+\pi^{\frac{1}{2}} \Gamma\left(s-\frac{1}{2}\right) a^{\frac{1}{2}-s} / 2 \Gamma(s)+\frac{2 \pi^{s}}{\Gamma(s)} \Sigma\left(\frac{a}{n^{2}}\right)^{\left(\frac{1}{2}-s\right) / 2} K_{\frac{1}{2}-s}(2 \pi n \sqrt{a}) .
$$

If $s=1$, we find by using (2.3) and (2.4) that

$$
\Sigma \frac{1}{n^{2}+a}=-\frac{1}{2 a}+\frac{\pi}{a^{\frac{1}{2}}}\left(\frac{1}{2}+\frac{e^{-2 \pi \sqrt{a}}}{1-e^{-2 \pi \sqrt{a}}}\right)
$$

This result is, of course, known. Also $f(-n, a)=-a^{n} / 2$, where $n$ is a nonnegative integer, and $f(s, a)$ has poles at $s=\frac{1}{2},-\frac{1}{2},-\frac{3}{2}, \ldots$ 


\title{
DIRICHLET SERIES AND HECKE'S FUNCTIONAL EQUATION 313
}

Next, consider $f(s)=\Sigma \tau(n) n^{-s}$, where $\tau(n)$ denotes Ramanujan's arithmetical function. It is well known that $f(s)$ is entire and of signature $(1,12,1)$. By corollary 1 ,

$$
f(s, a)=\frac{2(2 \pi)^{s}}{\Gamma(s)} \Sigma \tau(n)\left(\frac{a}{n}\right)^{(12-s) / 2} K_{12-s}(4 \pi \sqrt{a n}) .
$$

$f(s, a)$ is entire and has simple zeros at $s=0,-1,-2, \ldots$.

\section{REFERENCES}

(1) K. Chandrasekharan and Raghavan Narastmhan, Hecke's functional equation and arithmetical identities, Ann. of Math. 74 (1961), 1-23.

(2) Erich Hecke, Dirichlet Series, planographed lecture notes, Princeton Institute for Advanced Study, Edwards Brothers, Ann Arbor, 1938.

(3) J. E. LrtTLEwood, Lectures on the Theory of Functions, Oxford University Press, 1944.

(4) E. C. Titchmarsh, Theory of Fourier Integrals, 2nd ed., Clarendon Press, Oxford, 1948.

(5) G. N. Watson, Theory of Bessel Functions, 2nd ed., University Press, Cambridge, 1944.

\author{
UNIVERSITY OF GLASGOW \\ GlasGow, W.2 \\ AND \\ UNIVERSITY OF ILLINOIS \\ URBANA, ILLINOIS
}

